doi: 10.1111/ajd.12236

Dear Editor,

\section{Verrucous melanoma simulating melanoacanthoma: Dermoscopic, reflectance confocal microscopic and high-definition optical coherence tomography presentation of a rare melanoma variant}

Melanoma is characterised by a variety of clinical and histopathological presentations. ${ }^{1,2}$ Dermoscopy is a fast, non-invasive technique that increases diagnostic accuracy for both melanocytic and non-melanocytic skin tumours. One example of a potential pitfall in melanoma diagnosis using dermoscopy is represented by seborrheic keratosis (SK) that looks like melanomas, which may show overlapping features of both tumours. ${ }^{1}$

Reflectance confocal microscopy (RCM) and high-definition optical coherence tomography (HD-OCT) are non-in-
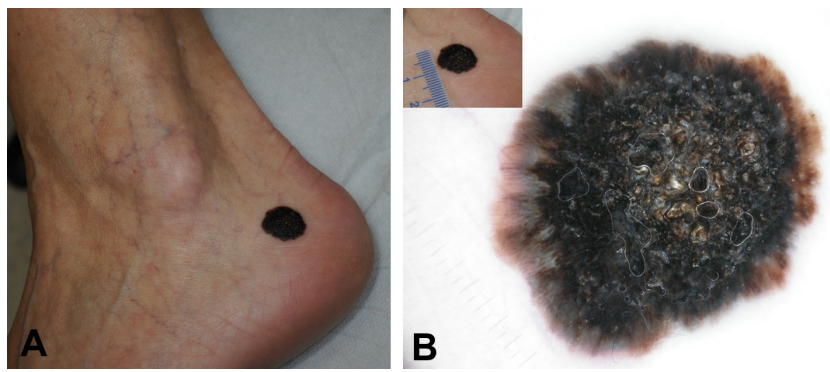

Figure 1 (a) Sharply demarcated, heavily pigmented black plaque with a verrucous surface on the left ankle. (b) Dermoscopy enabled the observation of peripheral streaks displaying variable nuances of blue and grey colouration arising from adjacent, confluent black, structureless areas surrounding a central aggregation of multiple, large, confluent comedo-like openings and keratotic plugs; a few milia-like cysts were also noted. vasive imaging techniques that are considered second or tertiary level diagnostic tools for the diagnosis of clinically and dermoscopically selected equivocal skin lesions.

Herein we report the case of a 56-year-old Caucasian woman who presented with a 12-month history of a new, growing, asymptomatic, pigmented plaque on her left ankle. A physical examination disclosed a sharply demarcated, heavily pigmented black plaque with a verrucous surface and a maximum diameter of $2 \mathrm{~cm}$ (Fig. 1a). Based on the clinical history and dermoscopic features (details in Fig. 1b) a diagnosis of verrucous melanoma versus SK, melanoacanthoma type, was made. The lesion was further examined by RCM and HD-OCT (details in Figs 2,3) that favoured the diagnosis of melanoma. Therefore, surgical excision of the lesion with narrow margins was performed. A histopathological examination (details in Fig. 4) showed features of an invasive melanoma, verrucous variant.

From a histopathological perspective, a melanoma clinically resembling SK could correspond to three rare variants: verrucous-hyperkeratotic melanoma, follicular melanoma and discohesive (acantholytic-like) melanoma. ${ }^{2-4}$ Kuehnl-Petzoldt and colleagues ${ }^{3}$ described a series of the verrucous-hyperkeratotic variant of melanoma, showing marked verrucous hyperplasia and hyperkeratosis. To date, only rare additional cases of its clinical and histopathological presentation have been reported.

Melanoacanthoma is a rare, heavily pigmented variant of SK showing a marked increase in the concentration of melanocytes. Our case could be clinically mistaken for melanoacanthoma due to the presence of a sharply demarcated, hyperpigmented plaque with a verrucous surface. However, the clinical history and unusual location for a SK raised doubts about the diagnosis. Moreover, dermoscopy disclosed equivocal structures of peripheral streaks arising from a heavily pigmented centre, which are commonly
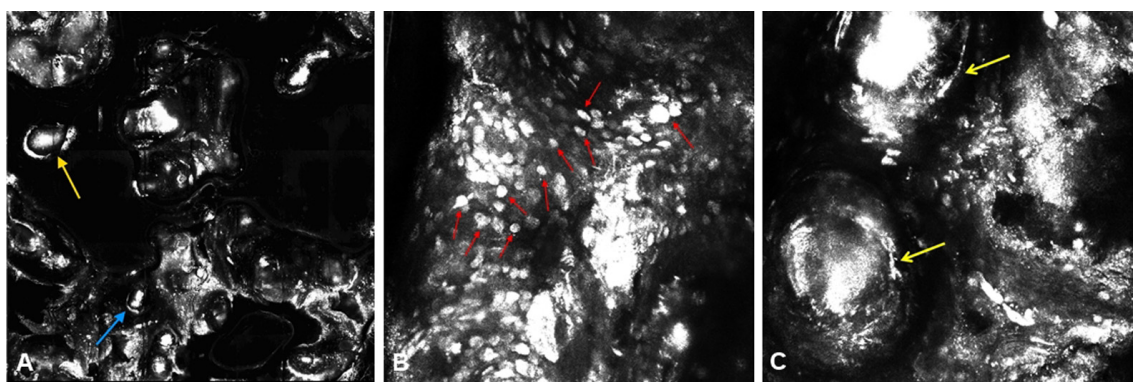

Figure 2 Reflectance confocal microscopy (RCM) evaluation revealed large, roundish, pleomorphic pagetoid cells showing a hyporeflective nucleus and bright cytoplasm, both isolated or focally aggregated forming irregular nests (red arrows) within a hyperkeratotic epidermis with a disarrayed pattern; intraepidermal keratin-filled invaginations (blue arrow) and bright reflecting horn cysts (yellow arrows) were additionally seen correlating to the dermoscopic findings of comedo-like openings and milia-like cysts, respectively; elongated cords, bulbous projections and well-defined papillae were absent. (a) RCM mosaic $4 \times 4 \mathrm{~mm}$. (b) RCM basic image $0.5 \times 0.5 \mathrm{~mm}$. (c) RCM basic image $0.5 \times 0.5 \mathrm{~mm}$.

Abbreviations:

\begin{tabular}{ll}
\hline HD-OCT & high-definition optical coherence tomography \\
SK & seborrheic keratosis \\
RCM & reflectance confocal microscopy
\end{tabular}



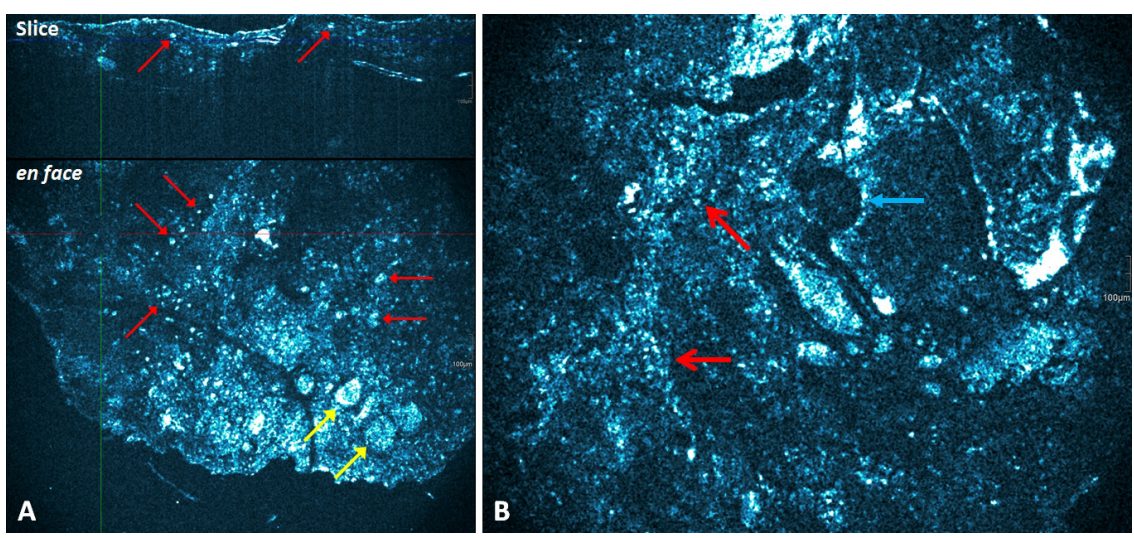

Figure 3 (a) High-definition optical coherence tomography examination showed a loss of normal epidermal and dermal layering in both slice and en face modes, with hyperkeratosis and acanthosis; the dermal-epidermal junction was not seen in the cross-sectional mode, where atypical melanocytes (red arrows) could be observed in the upper part of the acanthotic epidermis; an epidermal disarray was seen in en face imaging together with large atypical melanocytes (red arrows) with abundant reflective cytoplasm and hyporeflective nucleus relating to a pagetoid spread; junctional melanocytic aggregates distorted rete ridges resulting in inhomogeneous loose junctional nests with atypical melanocytes; intraepidermal bright horn cysts were observed; (b) together with pseudofollicular openings (blue arrow).

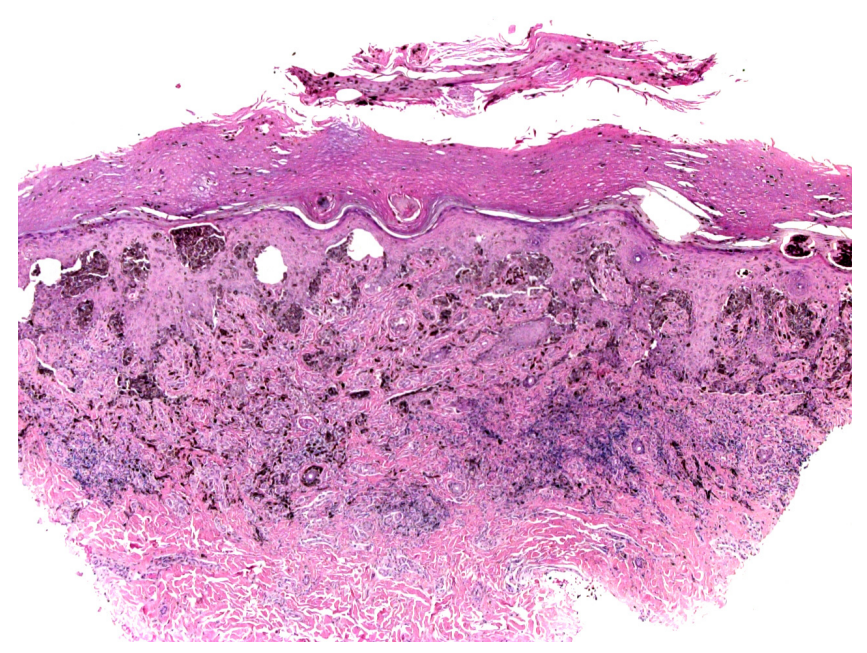

Figure 4 A histopathological examination showed atypical melanocytes arranged mainly in single units and atypical nests in all layers of the epidermis and in the papillary dermis; the epidermis showed prominent hyperplasia and hyperkeratosis: these features allowed the diagnosis of an invasive, verrucous melanoma with a tumour thickness of $0.9 \mathrm{~mm}$ and less than 1 mitosis per $\mathrm{mm}^{2}$ $(\mathrm{H} \& \mathrm{E} \times 40)$.

seen in pigmented Spitz naevus but have been also described in melanoma and in a case of melanoacanthoma. ${ }^{5}$ On the other hand, multiple, confluent and keratotic plugs were additionally present. They are common features in SK but can be regarded as unusual findings in melanocytic tumours.

To date, reports about the RCM and HD-OCT patterns of verrucous melanoma are lacking. To the best of our knowledge this is the first report about the correlation between the dermoscopic, RCM, HD-OCT and histopathological features of verrucous melanoma. Our case high- lights the fact that RCM and HD-OCT may complement and aid the sometimes difficult clinical and dermoscopic diagnosis of a SK that looks like a melanoma. These non-invasive techniques allow real-time and fast imaging acquisition with cellular resolution. Although with lesser resolution, HD-OCT enables an in vivo examination of the skin to a greater depth than RCM, reaching reticular dermis. The integration of all clinical and imaging data is of greatest importance to avoid misdiagnoses and the inappropriate treatment of melanomas mimicking SK.

André Oliveira, ${ }^{1}$ Edith Arzberger, ${ }^{2}$ Cesare Massone, ${ }^{1}$ Cristina Carrera ${ }^{3}$ and Iris Zalaudek ${ }^{2}$ ${ }^{1}$ Department of Dermatology, Hospital de Curry Cabral, Centro Hospitalar de Lisboa Central, Lisbon, Portugal,

${ }^{2}$ Department of Dermatology, Medical University of Graz, Graz, Austria and ${ }^{3}$ Dermatology Department, Hospital Clínic de Barcelona, Spain

\section{REFERENCES}

1. Argenziano G, Rossiello L, Scalvenzi M et al. Melanoma simulating seborrheic keratosis: a major dermoscopy pitfall. Arch. Dermatol. 2003; 139: 389-91.

2. Carrera C, Segura S, Palou J et al. Seborrheic keratosis like melanoma with folliculotropism. Arch. Dermatol. 2007; 143: 373-6.

3. Kuehnl-Petzcoldt C, Berger H, Wiebelt H. Verrucous-keratotic variations of malignant melanoma: a clinicopathological study. Am. J. Dermatopathol. 1982; 4: 403-10.

4. Ohnishi T, Hamano M, Watanabe S. Clinically verrucous and histologically discohesive melanoma. A case report with dermoscopic and immunohistochemical observations. Australas. J. Dermatol. 2014; 55: e21-23.

5. Rossiello L, Zalaudek I, Ferrara G et al. Melanoacanthoma simulating pigmented spitz nevus: an unusual dermoscopy pitfall. Dermatol. Surg. 2006; 32: 735-7. 